\title{
Acquired hemophilia A (AHA): underreported, underdiagnosed, undertreated medical condition
}

\author{
Doaa M. El Demerdash* ${ }^{*}$, Alia Ayad and Noha Tawfik
}

\begin{abstract}
Background: Acquired hemophilia A (AHA) is a rare acquired bleeding disorder occurred due to the formation of inhibitory antibodies neutralizing endogenous factor VIII.

Main body: About half the cases are idiopathic. Symptoms include severe and unexpected bleeding that could be life-threatening. High index of suspicion should be raised when unexplained subcutaneous or post-surgical bleeding with isolated prolonged APTT.

Conclusions: Acquired hemophilia $\mathrm{A}$ is a rare underdiagnosed underreported acquired hemostatic disorder that presents with sudden usually life-threatening bleeding; it is crucial to raise awareness and suspicion index of clinicians for early diagnosis and treatment to avoid morbidity and mortality.
\end{abstract}

Keywords: Hemophilia, Acquired, Bleeding, Prolonged APTT

\section{Background}

Acquired hemophilia A (AHA) is a rare bleeding disorder; it is an underreported condition because of the severity of AHA at presentation with life-threatening bleeding especially in the elderly in whom other bleeding conditions may coexist; also, it is underdiagnosed due to rarity and the unawareness of the condition which sometimes presented to surgical wards, and this may lead to delayed diagnosis hence worse outcome [1]. Our aim in this review article is to raise the awareness of the underreported acquired cause of bleeding.

\section{Main text}

\section{Acquired hemophilia recurrent scenario}

To clarify this recurrent scenario here, one of our cases, a female patient, 32 years old, G2P1, presented to the obstetric department for normal vaginal delivery without any previous known medical problems. She had

\footnotetext{
${ }^{*}$ Correspondence: dr_eldemerdash@kasralainy.edu.eg Internal Medicine Department, Kasr Al-Ainy Hospital, Faculty of Medicine, Cairo University, Cairo, Egypt
}

hemoglobin $(\mathrm{Hb} \%)$ of $11 \mathrm{~g} / \mathrm{dl}$ with a normal baseline coagulation profile. Her puerperium was complicated by excessive vaginal bleeding with a drop in her hemoglobin from 11 to $6.8 \mathrm{~g} / \mathrm{dl}$. Hysterectomy was performed for the persistent vaginal bleeding that was complicated by intra-abdominal hemorrhage. Her condition continued to deteriorate; the gynecologist feels that something was wrong with no obvious obstetric cause of bleeding, and she was referred to ICU with a diagnosis of DIC. Her labs on admission are summarized in Table 1; the striking result noticed was an isolated prolonged APTT. The striking result noticed was an isolated prolonged aPTT, with normal platelets count, normal PT, and slightly elevated d-dimer and FDP, and that is why the diagnosis of DIC was not convincing

Acquired hemophilia A (AHA) which is a severe acquired rare bleeding disorder. It is a rare bleeding disorder; in the reported cases, only 1-1.5 per million persons are affected yearly [2], AHA characterized by suddenly appearing autoantibodies (inhibitors) that partially or completely neutralize the activation or function or accelerate the clearance of factor VIII [3]. 
Table 1 Initial laboratory workup of an acquired hemophilia case

\begin{tabular}{lll}
\hline Laboratory tests & Patient's value & Normal value \\
\hline PC & $80 \%$ & $70-100 \%$ \\
Platelet count & $262,000 \mathrm{~mm}^{3}$ & $130-400 \mathrm{~mm}^{3}$ \\
aPTT & $68.4 \mathrm{~s}$ & $25.1-34.7 \mathrm{~s}$ \\
FDP & $20 \mathrm{mg} / \mathrm{L}$ & $<10 \mathrm{mg} / \mathrm{L}$ \\
D-Dimer & $1 \mu \mathrm{g} / \mathrm{mL}$ & $<0.5 \mu \mathrm{g} / \mathrm{mL}$ \\
\hline
\end{tabular}

Although it is like well-known congenital hemophilia in lacking factor VIII activity and hence bleeding tendency, but it is totally different from the well-known congenital hemophilia as It affects both males and females without previous family or personal history of bleeding while congenital hemophilia affects only males with a long history or family history of bleeding [4].

AHA incidence is typically observed in old age (> 60 years old) but another peak is noticed in middle age females especially with reported postpartum AHA cases [5].

Etiology is unknown in half of the reported cases but reported associated conditions (Table 2) in the other half were solid and hematological malignancies, autoimmune conditions such as SLE, dermatological conditions as pymphygus, drug-induced, pregnancy, and recently reported with COVID-19 and post COVID vaccines $[11,12]$.

Table 2 Conditions may be associated with acquired hemophilia A

\section{Conditions may be associated with acquired hemophilia $A$}

Malignancies [7]

\section{Dermatologic disorders [8]}

\section{Allergic drug reactions [9]}

Postpartum AHA [10]

Other conditions
Rheumatoid arthritis

Systemic lupus erythematosus

Multiple sclerosis

Temporal arteritis

Sjögren syndrome

Autoimmune hemolytic anemia

Goodpasture syndrome

Myasthenia gravis

Graves disease

Autoimmune hypothyroidism

\section{Solid tumors}

(prostate, lung, colon, pancreas, stomach, bile duct, head and neck, cervix, breast, melanoma, kidney)

Hematologic malignancies

Chronic lymphocytic leukemia

Non-Hodgkin lymphoma

Multiple myeloma

Waldenström macroglobulinemia

Myelodysplastic syndrome

Myelofibrosis

AML (M6)

Bullous Pemphigoid

Psoriasis vulgaris

Vitiligo

Squamous cell carcinoma

Penicillin and its derivatives

Sulfamides

Phenytoin

Chloramphenicol

Methyldopa

Depot thioxanthene

Interferon alfa

Fludarabine

Bacille Calmette-Guérin (BCG) vaccination

Desvenlafaxine

Inflammatory bowel disease, ulcerative colitis

Respiratory diseases (e.g., asthma, chronic obstructive pulmonary disease) Diabetes

Acute hepatitis B infection

Acute hepatitis C infection 
The cause of bleeding tendency is the same in both classic and AHA which decrease in the factor VIII activity. However, the clinical manifestation is not identical; in classic congenital hemophilia, usually, spontaneous bleeding into joints is typical, while in AHA, type of bleeding is different which is massive subcutaneous blood extravasations and mucosal hemorrhages [13].

The bleeding phenotype in AHA is variable ranging from mild to life-threatening bleeding, and there is poor correlation between FVIII level and inhibitor titer at presentation with bleeding severity unlike the congenital hemophilia bleeding phenotype [14].

The mortality rate is $41 \%$ if AHA patients are not treated: within the 1st week of presentation, mortality occurs due to GIT and lung bleeding Later, mortalities are usually from intracranial and retroperitoneal hemorrhages [15].

It is worth to be noted that $10 \%$ of patients do not present with bleeding and accidentally discovered with laboratory workup; therefore, a prolonged APTT should never be ignored prior to invasive procedures [16].

\section{Post-partum acquired hemophilia A}

We must focus on the entity which is postpartum acquired hemophilia in which there is spontaneous development of autoantibodies (inhibitors) against factor VIII during the peripartum period; it is reported in $7-21 \%$ of AHA cases [10]. Documented cases of postpartum AHA in EACH2 registry which included 501 AHA cases from 117 hemophilia centers in 13 European countries was $42(8.4 \%)$. Mortality due to hemorrhage in these cases varies between $12 \& 22 \%$ [17]. It Should be suspected in postpartum or pregnant patients who hemorrhage for no apparent cause and who have no previous history of bleeding disorders. Post-partum AHA usually appear after the 1 st pregnancy in $80 \%$ of the cases. Most reported cases arise from 1 to 4 months after delivery, but some cases presented up to 1 year after delivery [18].

It commonly presents as severe ecchymosis, soft tissue hematomas, and severe life-threatening hemorrhage. Vaginal bleeding could be the presenting symptom if the inhibitor appears early in the course, so it is usually misdiagnosed by an obstetrician especially with no previous bleeding or family history in presented females with postpartum unexplained bleeding. Intraplacental transfer of antibodies and intracerebral hemorrhage in neonates are reported in few cases [19].

\section{AHA laboratory workup}

Laboratory workup of a suspected case of AHA especially in the setting of unexplained bleeding associate with an isolated prolonged aPTT without previous medical or family history of bleeding should include measuring factor VIII activity and its inhibitor in those patients.

\section{Initial workup}

Individuals present with new-onset bleeding without previous history of bleeding disorders. Labs will show an isolated increase in aPTT with a normal PT, platelet count, and thrombin time. This points to either a deficient factor VIII or the presence of a factor VIII Inhibitor.

Therefore, a mixing study must be ordered to determine the cause. If there is a factor deficiency, the results will show a correction of PTT. If there is a factor inhibitor present, the PTT will remain elevated and uncorrected or partially corrected [2].

\section{Confirmation test}

Factor VIII activity test to exclude the presence of antiphospholipid antibodies and Bethesda assay to measure the strength of the inhibitors in the plasma, expressed in Bethesda units [20]. Workup of a case with AHA is summarized in Fig. 1.

\section{Why acquired hemophilia $A$ is undermanaged?}

AHA is a rare but potentially lethal disease this make it underreported, no comparative clinical studies are available in AHA, the severity of the clinical condition of patients at presentation, and the decisions are often based on the clinical experience of treating physicians, all these factors lead to undermanagement of AHA [21].

Management of AHA is representing a medical challenge from its diagnosis to its treatment, the early initiation of Therapy results in higher efficacy. The only parameter that differed between patients who responded to treatment and those who did not was a delay in time to treatment [22].

Optimal management included the use of "bypass" agents when active bleeding presents, altogether with active inhibitor eradication through immunosuppressant drugs, the 2 lines of treatment should be started aside with most importantly treatment of any underlying condition, e.g., autoimmune disease. Further recommendations should include avoidance of invasive procedures that may induce bleeds even sampling of blood or inserting IV cannula [23].

\section{Control bleeding if present}

Lines to control the acute bleeding includes:

- Bypassing agents: Recombinant Factor VIla (rFVIIa) or FEIBA, activated prothrombin complex concentrate (APCC) 


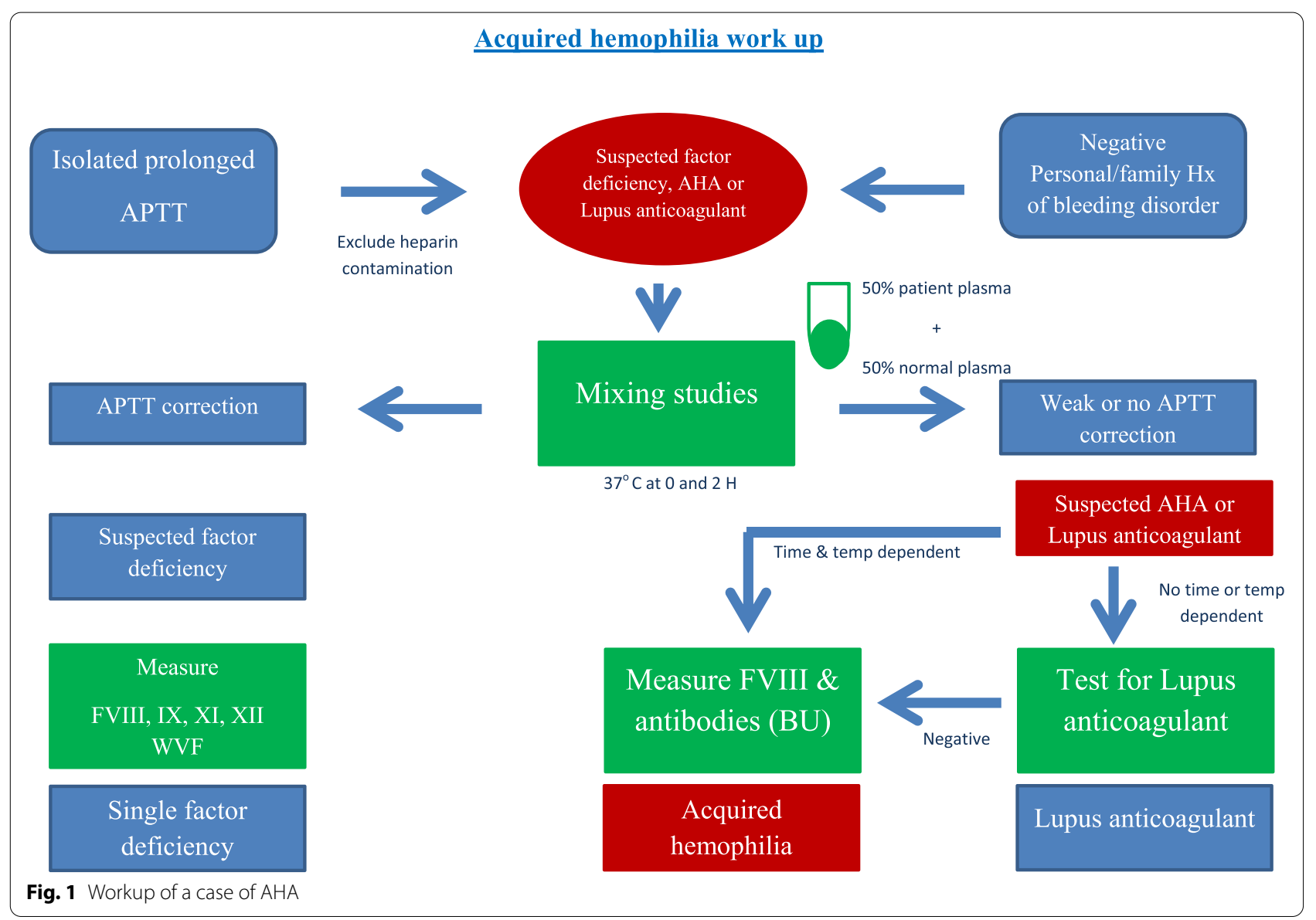

- Recombinant porcine factor VIII rpFVIII (susoctocog alfa)

Registries data did not show a clear efficacy or safety benefit of one drug over the others.

\section{- Recombinant Factor VIla (rFVIIa)}

It is given as $90 \mu \mathrm{g} / \mathrm{kg}$ bolus at every $2 \mathrm{~h}$, Cases of mild bleeding, one or two doses of rFVIIa may be sufficient. Once hemostasis has been achieved, the dose interval can be increased successively to every $4,6,8$, or $12 \mathrm{~h}$ when indicated. rFVIIa controls bleeding at the site of vascular injury only. Its safety profile includes that thrombotic events of rFVIIa $<1 \%$ with an $\sim 800,000$ doses, it is not contraindicated for use with antifibrinolytics, it is a pure FVII, with no risk of anamnesis, and also it is a recombinant product with no risk of viral transmission [24].

- FEIBA, an activated prothrombin complex concentrate (aPCC)
It includes factor II, VII, IX, X, and small amount of factor VIIIa. Also, it includes natural anticoagulant (protein $\mathrm{C}$ and $\mathrm{S}$ ) in physiological balance. aPCC doses in the range of $50-100 / \mathrm{kg}$ units every $8-12 \mathrm{~h}$ are given by intravenous infusion. it is important not to exceed a total of 200 units $/ \mathrm{kg}$ within a 24 -h period as this may be associated with a risk of VTE or DIC. We must point out that tranexamic acid should not be given together with this agent $[25,26]$.

- Recombinant porcine factor VIII rpFVIII (susoctocog alfa)

It could be given in cases of AHA as human anti-FVIII autoantibodies have low cross-reactivity with porcine FVIII, it could be given in Patients with AHA with a serious bleed but it should be excluded if they had an antirpFVIII inhibitor titer $>20$ BU.

rpFVIII dose of $200 \mathrm{U} / \mathrm{kg}$, followed by further doses to maintain trough levels of factor VIII $>50 \%$.close monitoring of FVIII activity during therapy is recommended to avoid the theoretical risk of thrombosis while there were no thromboembolic events reported [27]. 
Table 3 Immunosuppressive therapy for AHA

\begin{tabular}{ll}
\hline FVIII $\geq 1 \%$ and $<20 \mathrm{BU} / \mathrm{ml}$ & Steroids alone \\
$\mathrm{FVIII}<1 \%$ or $>\mathbf{2 0 ~ B U / m I}$ & $3-4$ weeks \\
& If no response add cyclophosphamide or rituximab \\
& Steroids and cyclophosphamide or rituximab 3-4 weeks \\
& If no response add cyclophosphamide or rituximab
\end{tabular}

\section{Eradications of inhibitors by immunosuppressants}

In all patients with AHA, they should receive immunosuppressants to eradicate inhibitors in form of corticosteroids alone or plus either rituximab or cytotoxic agents, while speaking about steroid therapy mentions that oral prednisone is the preferred one [28]. If FVIII $\geq 1 \mathrm{IU} / \mathrm{dL}$ and inhibitor titer $\leq 20 \mathrm{BU}$ at baseline receive 1st line treatment with corticosteroids alone for 3-4 weeks is recommended, but If FVIII $<1 \mathrm{IU} / \mathrm{dL}$ or inhibitor titer $>20 \mathrm{BU}$ it is suggested combining corticosteroids with rituximab or a cytotoxic agent for 1st line therapy. If no response to $1^{\text {st }}$ line therapy 2 nd line therapy with rituximab or a cytotoxic agent, whichever was not used during first-line therapy is suggested, immune tolerance induction in AHA. Or high-dose intravenous immunoglobulins for inhibitor eradication in patients with AHA are not recommended [21].

Because relapse has been reported in approximately 1 in 5 patients after immunosuppressive therapy is discontinued. The assessment of the response requires follow-up of factor VIII activity and inhibitors assay. It is recommended of using FVIII:C monitoring monthly during the first 6 months, every 2-3 months up to 12 months, and every 6 months during the second year and beyond, if possible [21] (Table 3).

Table 2 summarizes 2020 International recommendations of immunosuppressive therapy in the treatment of acquired hemophilia A.

\section{Back to presented scenario}

workup of the patient was continued as a case presented with isolated prolonged aPTT. Laboratory results confirm the diagnosis of postpartum AHA as demonstrated below.

Table 4 Continued laboratory workup of an acquired hemophilia case

\begin{tabular}{lll}
\hline aPTT Mixing studies & $\mathbf{6 7} \mathbf{~ s}$ & $\mathbf{2 5 . 1 - 3 4 . 7 ~ s}$ \\
\hline LA & Negative & Negative \\
Factor VIII rate & $4 \%$ & $70-150 \%$ \\
Factor VIII inhibitor level & $10 \mathrm{BU}$ & $<0.6 \mathrm{BU}$ \\
Factor IX rate & $103.9 \%$ & $70-120 \%$ \\
von Willebrand factor & $89.5 \%$ & $50-160 \%$ \\
\hline
\end{tabular}

Diagnosis of postpartum AHA was confirmed by doing mixing studies for aPTT which was not corrected by adding normal plasma which confirm the presence with of inhibitor, absence of antibodies for antiphospholipid altogether with a reduced level of factor VIII, and presence of FVIII antibodies in our case confirm the final diagnosis of postpartum AHA (Table 4).

Her bleeding was life-threatening and massive due to late diagnosis and management, also it was aggravated by the surgical intervention (hysterectomy), as we must point out that any surgical intervention may aggravate antibodies formation and hence the severe bleeding [28].

To stop bleeding, rFVIIa was given $90 \mathrm{mic} / \mathrm{kg}$ every 2 $\mathrm{h}$ then gradually spaced to be every 6 h, 12 h, and 24 h (12 doses were needed till regression), prednisone $1 \mathrm{mg} / \mathrm{kg} /$ day was added (4 weeks duration) to eradicate inhibitor, her course was stable apart from intermittent symptoms and prolongation of APTT which mean persistent inhibitor rituximab was added in a dose of $375 \mathrm{mg} / \mathrm{m}^{2}$ for 4 doses, and resolution of inhibitors was achieved after 3 weeks of initiation of added rituximab.

\section{Conclusions}

Acquired hemophilia A is a rare underdiagnosed underreported acquired hemostatic disorder that presents with sudden usually life-threatening bleeding, it is crucial to raise awareness and suspicion index of clinicians for early diagnosis and treatment to avoid morbidity and mortality.

\section{Abbreviations}

AHA: Acquired hemophilia A; APCC: Activated prothrombin complex concentrate; APTT: Activated partial thromboplastin time; BCG: Bacille CalmetteGuerin; BU: Bethesda unit; COVID: Coronavirus sisease; DIC: Disseminated intravascular coagulation; EACH2: European Acquired Hemophilia Registry; FDP: Fibrin degradation products; FEIBA: Factor eight inhibitor bypass activity; GIT: Gastrointestinal tract; ICU: Intensive care unit; rPFVIII: Recombinant porcine factor VIII; SLE: Systemic lupus erythematosus; VTE: Venous thromboembolism.

\section{Acknowledgements \\ Not applicable}

Authors' contributions

Written by DM, and all work revised by DM, AA, and NT. All authors have read and approved the manuscript.

Funding

Self-funded 
Availability of data and materials

Not applicable

\section{Declarations}

Ethics approval and consent to participate

Not applicable

\section{Consent for publication}

Not applicable

\section{Competing interests}

The authors declare that they have no competing interests.

Received: 11 July 2021 Accepted: 28 September 2021

Published online: 25 January 2022

\section{References}

1. Singh N, Singh Lubana S, Dabrowski L (2020) Acquired hemophilia A: a potentially fatal bleeding disorder. Cureus. 12(6):e8744

2. Huth-Kühne A, Baudo F, Collins P et al (2009) International recommendations on the diagnosis and treatment of patients with acquired hemophilia A. Haematologica. 94:566-575

3. Franchini M, Lippi G (2011) Acquired hemophilia A. Adv Clin Chem 54:71-80

4. Bouvry P, Recloux P (1994) Acquired hemophilia. Haematologica 79:550-556

5. Kessler CM, Ma AD, Al-Mondhiry HA, Gut RZ, Cooper DL (2016) Assessment of acquired hemophilia patient demographics in the United States: the Hemostasis and Thrombosis Research Society Registry. Blood Coagul Fibrinolysis 27(7):761-769

6. Kessler CM, Knöbl P (2015) Acquired haemophilia: an overview for clinical practice. Eur J Haematol 95(Suppl 81):36-44

7. Reeves BN, Key NS (2012) Acquired hemophilia in malignancy. Thromb Res 129(Suppl 1):S66-S68

8. Binet Q, Lambert C, Sacré L, Eeckhoudt S, Hermans C (2017) Successful management of acquired hemophilia A associated with bullous pemphigoid: a case report and review of the literature. Case Rep Hematol 2017:2057019

9. Franchini M, Capra F, Nicolini N, Veneri D, Manzato F, Baudo F, Lippi G (2007) Drug-induced anti-factor VIII antibodies: a systematic review. Med Sci Monit 13(4):RA55-RA61

10. Karakuş V, Celik M, Soysal D, Payzın B (2012) Postpartum acquired hemophilia factor VIII inhibitors and response to therapy. Turk J Haematol 29(2):197-198. https://doi.org/10.5505/tjh.2012.68725

11. Collins P (2011) Management of acquired haemophilia A. J Thromb Haemost 9:226-235

12. Radwi M, Farsi S (2021 Jun) A case report of acquired hemophilia following COVID-19 vaccine. J Thromb Haemost 19(6):1515-1518

13. Collins P, Hirsch S, Baglin T et al (2007) Acquired hemophilia A in the United Kingdom: a 2-year national surveillance study by the United Kingdom Haemophilia Centre Doctors' organization. Blood. 109:1870-1877

14. Collins P, Baudo F, Huth-Kühne A et al (2010) Consensus recommendations for the diagnosis and treatment of acquired hemophilia A. BMC Res Notes 3:161

15. Sborov DW, Rodgers GM (2012) Acquired hemophilia A: a current review of autoantibody disease. Clin Adv Hematol Oncol 10(1):19-27

16. Webert KE (2012) Acquired hemophilia A. Semin Thromb Hemost 38(7):735-741

17. Knoebl P, Marco P, Baudo F, Collins P, Huth-Kühne A, Nemes L, Pellegrini F, Tengborn L, Lévesque H (2012 Apr) EACH2 Registry Contributors. Demographic and clinical data in acquired hemophilia $A$ : results from the European Acquired Haemophilia Registry (EACH2). J Thromb Haemost 10(4):622-631

18. Tengborn L, Baudo F, Huth-Kühne A, Knoebl P, Lévesque H, Marco P, Pellegrini F, Nemes L, Collins P (2012 Nov) EACH2 registry contributors. Pregnancy-associated acquired haemophilia A: results from the European Acquired Haemophilia (EACH2) registry. BJOG. 119(12):1529-1537
19. Azam K, Batool Z, Malik A, Chaudhry M, Abdullah M (2020) Postpartumacquired hemophilia A presenting as hemoperitoneum: a case report. Cureus. 12(12):e11817

20. Miller CH (2018) Laboratory testing for factor VIII and IX inhibitors in haemophilia: A review. Haemophilia 24(2):186-197.

21. Tiede A, Collins P, Knoebl P et al (2020) International recommendations on the diagnosis and treatment of acquired hemophilia A. Haematologica. 105(7):1791-1801

22. Baudo F, Collins P, Huth-Kuhne A (2012) Management of bleeding in acquired hemophilia A: results from the European Acquired Haemophilia (EACH2) Registry. Blood. 120(1):39-46

23. Knöbl P (2018) Prevention and management of bleeding episodes in patients with acquired hemophilia A. Drugs. 78(18):1861-1872

24. Sumner MJ, Geldziler BD, Pedersen M, Seremetis S (2007) Treatment of acquired haemophilia with recombinant activated FVII: a critical appraisal. Haemophilia. 13(5):451-461

25. Sallah S (2004) Treatment of acquired haemophilia with factor eight inhibitor bypassing activity. Haemophilia. 10(2):169-173

26. Ehrlich HJ, Henzl MJ, Gomperts ED (2002) Safety of factor VIII inhibitor bypass activity $\left(\right.$ FEIBA $\left.^{\circledR}\right)$ : 10-year compilation of thrombotic adverse events. Haemophilia. 8(2):83-90

27. Türkantoz H, Königs C, Knöbl P, Klamroth R, Holstein K, Huth-Kühne A, Heinz J, Eichler H, Tiede A (2020) Cross-reacting inhibitors against recombinant porcine factor VIII in acquired hemophilia A: Data from the GTH-AH 01/2010 Study. J Thromb Haemost 18(1):36-43

28. Pham TV, Sorenson CA, Nable JV (2014) Acquired factor VIII deficiency presenting with compartment syndrome. Am J Emerg Med 32(2):195

\section{Publisher's Note}

Springer Nature remains neutral with regard to jurisdictional claims in published maps and institutional affiliations.

\section{Submit your manuscript to a SpringerOpen ${ }^{\circ}$ journal and benefit from:}

- Convenient online submission

$\checkmark$ Rigorous peer review

- Open access: articles freely available online

- High visibility within the field

- Retaining the copyright to your article

Submit your next manuscript at $\boldsymbol{\nabla}$ springeropen.com 